\title{
Wearable Dual-band Sierpinski Fractal PIFA using Conductive Fabric
}

\author{
P.J. Soh, G.A.E. Vandenbosch, S.L Ooi and M.R.N Husna
}

A novel PIFA, incorporating a triangular Sierpinski gasket, is presented. The used conducting textile and polyester fleece allow easy on-body integration and simple practical fabrication. It shows dual-band response, combining a 16 $\%$ band at $2.45 \mathrm{GHz}$ with an upper $12 \%$ band at $5.2 \mathrm{GHz}$. The broadside gain is $3.1 \mathrm{dBi}$. Good agreement between calculated and measured data is observed.

Introduction: Antennas to be integrated on-body are crucial in catering for various future 802.15 wireless standards. The main barrier of implementing these antennas is usually the degraded performance when operating in proximity of the body, for example the reduced bandwidth. Large bandwidth or dual band behaviour is needed in multi-application wireless devices. Several techniques have been implemented to broaden the bandwidth, including the

introduction of slots [1, 2], folding and rolling up of larger elements [3, 4], addition of parasitic elements [5], and integration of several radiators into a single structure $[6,7]$. An effective way to generate dual- and multi-band frequency response is using fractal Sierpinski topologies. However, gasket monopoles, in which these structures are traditionally implemented, limit a planar implementation on a small physical area [8]. Incorporating the structure 
into a planar inverted-F antenna (PIFA), which is the key novelty in this letter, provides a means of planar implementation.

Topology: The PIFA incorporates a triangular Sierpinski radiator at the top, a rectangular ground plane at the bottom, both made from the conductive fabric Shieldlt ${ }^{\mathrm{TM}}$ from LessEMF Inc, USA, and a $6 \mathrm{~mm}$ thick layer of fleece in between. Top and bottom are connected by a shorting wall with a width $W_{s}$ of $4 \mathrm{~mm}$. The antenna is fed using an SMA connector at a position $\left(f_{\mathrm{V}}, f_{\mathrm{H}}\right)$. The topology is depicted in Fig. 1. The rip-stop fabric Shieldlt ${ }^{\mathrm{TM}}$ has a thickness $t$ of $0.17 \mathrm{~mm}$ and a surface resistance $R_{\mathrm{s}}$ of less than $0.05 \Omega / \mathrm{sq}$. It is derived from woven polyester, which is coated with copper and nickel. It comes with an adhesive backing which enables secure placement on the fleece without sewing. The prototype is benchmarked against a PIFA using a $0.035 \mathrm{~mm}$ thick copper tape, which also comes with an adhesive backing.

Design: At first the side length $s$ is estimated using conventional Sierpinski fractal calculation, as proposed in [9]

$$
s=\left\{\begin{array}{cc}
\frac{1}{\sqrt{3}}(0.3069+0.68 \rho x) \frac{c}{f_{c}}\left(\xi^{-1}\right)^{n} & \text { for } \mathrm{n}=0 \\
\frac{0.52}{\sqrt{3}} \frac{c}{f_{c}} \delta^{n}-\frac{h}{\sqrt{\varepsilon_{r}}} & \text { for } \mathrm{n}>0
\end{array}\right.
$$

where $n$ is the iteration number, $\rho=\xi-0.230735, x=1, c$ is the velocity of light in free space, $f_{c}$ is the desired center frequency, $\xi$ is the triangle's height ratio between two successive iterations $\left(\xi=D_{\text {iter(n) }} / D_{\text {iter(n+1) }}\right), \quad \delta$ is the scale factor, also given by $\delta=1 / \xi, h$ is the thickness and $\varepsilon_{\mathrm{r}}$ the relative permittivity of the substrate. Initial calculation using $\delta=2, f_{C}=2.45 \mathrm{GHz}, h=6 \mathrm{~mm}$, and $\varepsilon_{\mathrm{r}}=$ 
1.26 yields a zeroth iteration Sierpinski triangle with $s=35 \mathrm{~mm}$. This translates into a triangle height of $D=30 \mathrm{~mm}$ and a triangle base length $R_{\mathrm{W}}$ of $35 \mathrm{~mm}$.

The procedure to combine the Sierpinski triangle within a PIFA is started by simultaneously tuning the lower and upper band by properly choosing $D$. The upper frequency band $(5.2 \mathrm{GHz})$ is adjusted using $R \mathrm{w}, f_{\mathrm{H}}$, and $f_{\mathrm{V}}$, while the lower band $(2.45 \mathrm{GHz})$ is adjusted with $W_{s}$. Finally, overall fine-tuning of $S_{11}$ is achieved by modifying $G_{\llcorner}$. CST Microwave Studio is used as a computational tool in this optimization procedure. The final dimensions are $D=24 \mathrm{~mm} ; R \mathrm{w}=$ $34 \mathrm{~mm}, G_{\mathrm{L}}=44 \mathrm{~mm}, W_{s}=4 \mathrm{~mm}, f_{V}=9 \mathrm{~mm}$, and $f_{H}=8.5 \mathrm{~mm}$. The ground plane width $G_{\mathrm{W}}$ is chosen equal to $R_{\mathrm{W}}$ to ensure fabrication simplicity.

Results: The main challenge is to cater for possible frequency shift both at 2.45 and $5.2 \mathrm{GHz}$. This may occur due to the material inhomogeniety [10] and fabrication inaccuracy. Two Sierpinski fractal PIFAs, one using Shieldlt conductive textile and another using copper tape, were built and measured. Both reflection coefficients are shown in Fig. 2. Bandwidths of $570 \mathrm{MHz}$ (copper tape) and $345 \mathrm{MHz}$ (Shieldlt) are produced at $2.45 \mathrm{GHz}$, while bandwidths of $615 \mathrm{MHz}$ (copper tape) and $585 \mathrm{MHz}$ (Shieldlt) are obtained at 5.2 GHz. Comparing simulations with measurements for both prototypes; the frequency shift is higher at $2.45 \mathrm{GHz}$ compared to $5.2 \mathrm{GHz}$.

Measurements of the radiation patterns, gain, and efficiency were carried out in a Satimo SG-64 system. Simulated and measured radiation patterns show 
an excellent agreement in the main beam direction at both frequencies, see Fig. 3. Concerning efficiency, the agreement between measurements and simulations is better for the copper tape prototype. Simulations yield $93 \%$ at $2.45 \mathrm{GHz}$ and $89 \%$ at $5.2 \mathrm{GHz}$, while measurements show $86 \%$ and $78 \%$, respectively. For Shieldlt, simulations yield $90 \%$ at $2.45 \mathrm{GHz}$ and $93 \%$ at 5.2 GHz. Measurements yield $75 \%$ and $73 \%$, respectively. It is seen that at 2.45 $\mathrm{GHz}$ Shieldlt produces a measured gain of about $1 \mathrm{~dB}$ lower than copper tape (1.8 compared to $2.8 \mathrm{~dB}$ ). The difference is less in the $5.2 \mathrm{GHz}$ band (3.1 compared to $3.8 \mathrm{~dB}$ ). The gain measured at $5.2 \mathrm{GHz}$ is higher than at 2.45 $\mathrm{GHz}$. The difference between simulated and measured gain is higher for Shieldlt, about $1 \mathrm{~dB}$ at $2.45 \mathrm{GHz}$ and $0.5 \mathrm{~dB}$ at $5.2 \mathrm{GHz}$.

The lower conductivity of Shieldlt (about 200 times lower than for copper tape) explains the lower efficiency and gain. The larger differences between simulations and measurements for Shieldlt can be explained by the inherently lower fabrication accuracy because a textile is involved (compared to etching or milling processes), a possible discrepancy between the real material properties and the ones used in the simulations (quite complex materials are used, involving for example a polyester coating process). Resonance frequencies can also shift under slight bending and inconsistent substrate thickness conditions. This effect is unavoidable in case flexible materials like textiles are used.

Conclusion: A novel, dual-band planar inverted-F antenna (PIFA), based on the Sierpinski fractal gasket, is proposed and discussed. Its implementation 
using the conductive textile Shieldlt was proven to be feasible for wearable applications. Experimental data indicates that the antenna can be used both in the 2.45 and $5.2 \mathrm{GHz}$ bands. It has a compact antenna footprint and a performance comparable with conventional copper material. A monopole-like omni-directional radiation pattern is produced at broadside with a measured gain of about $3.1 \mathrm{dBi}$ and an efficiency of more than $70 \%$.

\section{References}

[1] MA, L., EDWARDS, R. M., BASHIR, S., and KHATTAK, M. I.: 'A wearable flexible multi-band antenna based on a square slotted printed monopole'. Loughborough Antennas and Propagation Conference, March 2008, Loughborough, pp. 345-348.

[2] SALONEN, P., JAEHOON, K., and RAHMAT-SAMII, Y.: 'Dual-band Eshaped patch wearable textile antenna'. Antennas and Propagation Society International Symposium, July 2005, Washington, pp. 466-469 Vol. $1 \mathrm{~A}$

[3] KABACIK, P., HOSSA, R., and BYNDAS, A.: 'Foldeable wideband small antenna element'. Antennas and Propagation Society International Symposium, June 2007, Hawaii USA, pp. 2598-2601.

[4] XINGYU, Z., and ANPING, Z.: 'Flexible compact planar inverted-F antenna for GSM/DCS/PCS triple-band applications'. Int Symp. in Antennas, Propagation and EM Theory, Nov 2008, Kunming, China, pp. $15-18$.

[5] QIANG, B., and LANGLEY R.: 'Wearable EBG antenna bending and crumpling'. Loughborough Antennas \& Propagation Conference, Nov 2009, Loughborough UK, pp. 201-204.

[6] GASPAR, D., and MOREIRA, A. A..'Belt antenna for wearable applications'. Antennas and Propagation Society International Symposium, June 2009, Charleston, USA , pp. 1-4.

[7] SANZ-IZQUIERDO, B., HUANG, F., and BATCHELOR, J. C.: 'Covert dual-band wearable button antenna'. Electronics Letters, 2006, 42, pp. 668-670.

[8] KRZYSZTOFIK, W. J.: 'Fractal antenna for WLAN/bluetooth multiplebands applications'. European Conf. on Antennas and Propagation, April 2009, Barcelona, pp. 2407-2410.

[9] MISHRA, R. K., GHATAK, R., and PODDAR, D.: 'Design Formula for Sierpinski Gasket Pre-Fractal Planar-Monopole Antennas [Antenna Designer's Notebook]'. Antennas and Propagation Magazine, IEEE, 2008, 50, pp. 104-107. 
[10] LOCHER, I., KLEMM, M., KIRSTEIN, T., and TROSTER, G.: 'Design and Characterization of Purely Textile Patch Antennas'. Advanced Packaging, IEEE Transactions on, 2006, 29, pp. 777-788.

\section{Authors' affiliations:}

P.J Soh and G.A.E Vandenbosch (ESAT-TELEMIC, Katholieke Universiteit Leuven, Kasteelpark Arenberg 10, 3001 Leuven, Belgium). Email: pingjack.soh@esat.kuleuven.be)

P.J Soh, S.L Ooi and M.R.N Husna (School of Computer and Communication, Universiti Malaysia Perlis (UniMAP), Block A, Seberang Ramai School Complex, No 12 and 14, Tmn Seberang Ramai Phase III, 02000 Kuala Perlis, Perlis, Malaysia)

S.L Ooi (Sulucon Research, 10760 NW 12th Drive, Plantation, Florida 33322, USA)

\section{Figure captions:}

Fig. 1 Antenna topology and dimensions; (a) top view, (b) perspective view, (c) fabricated prototype

Fig. 2 Measured and simulated reflection coefficient $\left(S_{11}\right)$ for both copper tape and Shieldlt conductive textile prototypes

Copper Tape PIFA simulated $S_{11}$

-o-o-o- Copper Tape PIFA measured $S_{11}$

---- Shieldlt Textile PIFA simulated $S_{11}$

- $\Delta$ - $\Delta$ - Shieldlt Textile PIFA measured $S_{11}$

Fig. 3. Measured and simulated radiation patterns ( $\mathrm{Phi}=0^{\circ}$ cut) (a) at 2.45 $\mathrm{GHz}$, and (b) at $5.2 \mathrm{GHz}$.

- - - Copper Tape simulated radiation pattern

-0-0-0- Copper Tape measured radiation pattern

Shieldlt Textile PIFA simulated radiation pattern

- $\Delta$ - $\Delta$ - Shieldlt Textile PIFA measured radiation pattern 
Figure 1
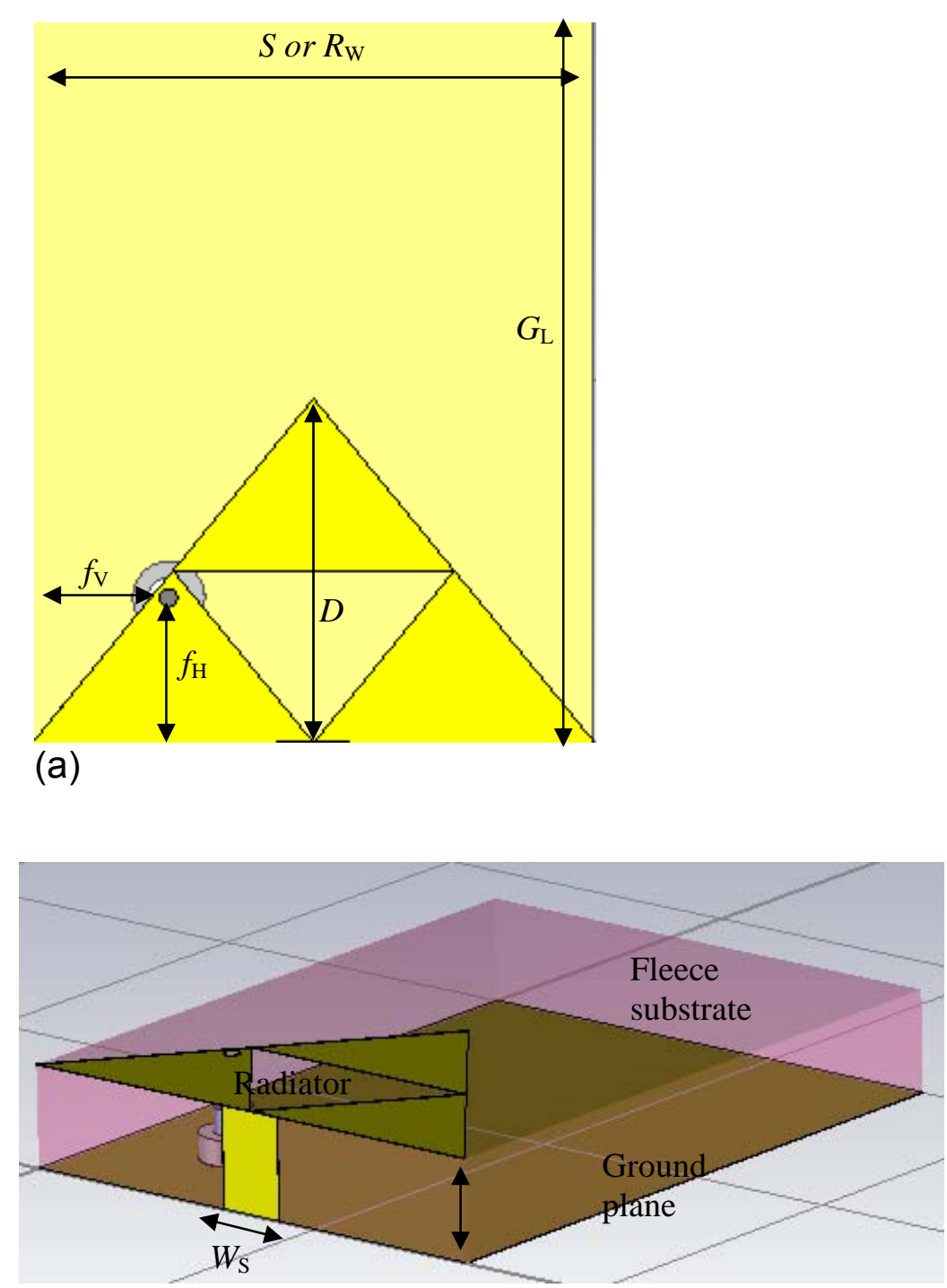

(b)

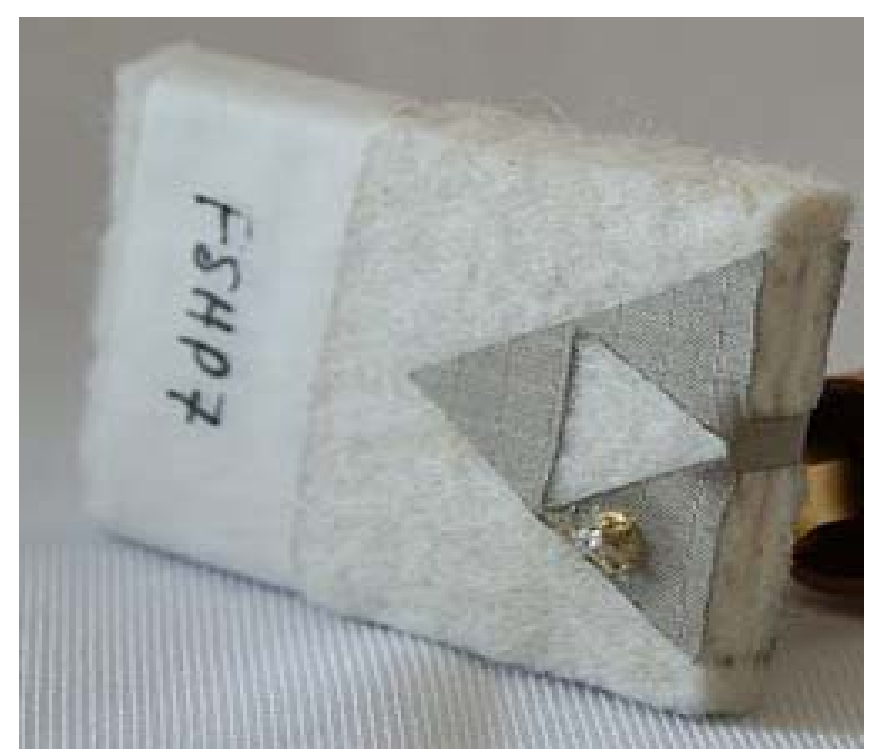

(c) 
Figure 2

Simulated and Measured S11 for Shieldlt and Coppertape PIFA Prototypes

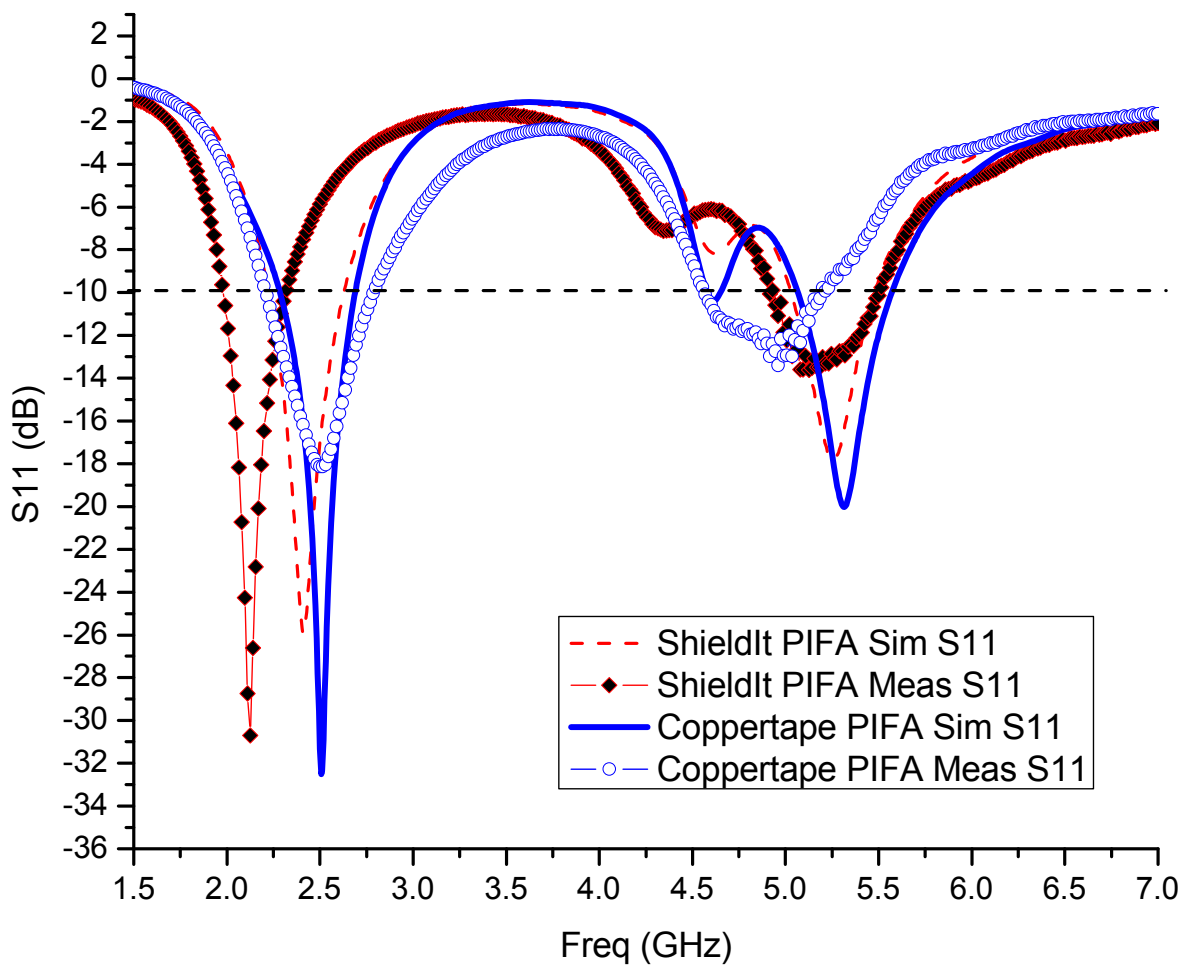


Figure 3

Meas \& Sim Rad Pattern (Phi=0 Cut) at $2.45 \mathrm{GHz}$ Meas \& Sim Rad Pattern (Phi=0 Cut) at 5.2 GHz

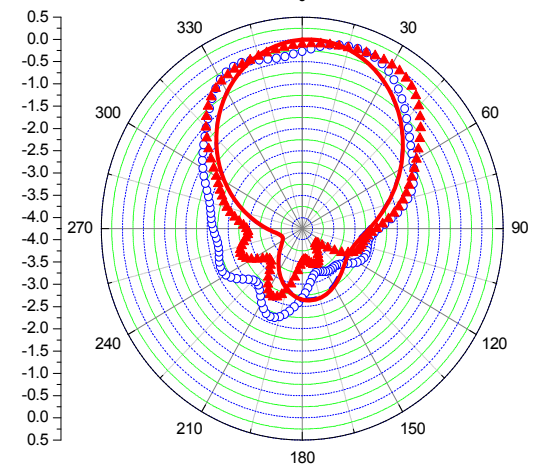

(a)

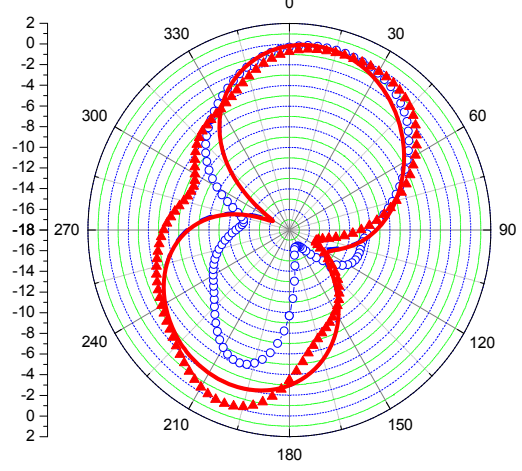

(b) 